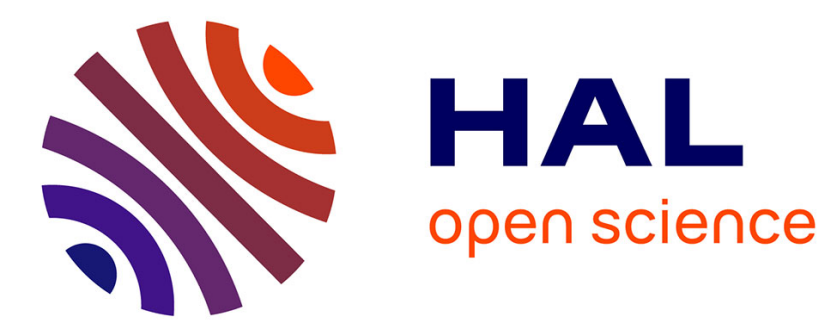

\title{
A propos de répétition : entre continuité et rupture Élisabeth Richard
}

\section{To cite this version:}

Élisabeth Richard. A propos de répétition: entre continuité et rupture. Semen - Revue de sémiolinguistique des textes et discours, 2015, 38, pp.95-114. hal-02025466

\section{HAL Id: hal-02025466 https://hal.univ-rennes2.fr/hal-02025466}

Submitted on 19 Feb 2019

HAL is a multi-disciplinary open access archive for the deposit and dissemination of scientific research documents, whether they are published or not. The documents may come from teaching and research institutions in France or abroad, or from public or private research centers.
L'archive ouverte pluridisciplinaire $\mathbf{H A L}$, est destinée au dépôt et à la diffusion de documents scientifiques de niveau recherche, publiés ou non, émanant des établissements d'enseignement et de recherche français ou étrangers, des laboratoires publics ou privés. 


\section{Semen}

Revue de sémio-linguistique des textes et discours

38 | 2015

Pragmatique de la répétition

\section{A propos de répétition : entre continuité et rupture}

Élisabeth Richard

\section{(2) OpenEdition}

Journals

Electronic version

URL: http://journals.openedition.org/semen/10323

ISSN: 1957-780X

Publisher

Presses universitaires de Franche-Comté

Brought to you by Université Rennes 2

Electronic reference

Élisabeth Richard, «A propos de répétition : entre continuité et rupture », Semen [Online], 38 | 2015, Online since 24 April 2015, connection on 19 February 2019. URL : http://journals.openedition.org/ semen/10323

This text was automatically generated on 19 February 2019

(c) Presses universitaires de Franche-Comté 


\title{
A propos de répétition : entre continuité et rupture
}

\author{
Élisabeth Richard
}

\section{La répétition chez un même locuteur}

\subsection{Répétition et mise en scène}

1 S'il est assez ordinaire, à l'oral comme à l'écrit, qu'un locuteur, heurté dans le qui-va-de-soi de son énonciation (J. Authier-Revuz), revienne sur un mot pour mieux le circonscrire ou le commenter, comme dans l'exemple (1), l'annuler, comme en (2), ou le renvoyer à un Autre, comme en (3) par exemple :

(1) Tout au long de son exposé - un exposé plutôt scabreux - M. Innocento n'eut qu'à se louer de son interlocuteur. [P. Benoît, Feux d'artifice à Zanzibar : 56]

(2) J'aime, que dis-je aimer ? j'idolâtre Junie [Racine, Britannicus]

(3) Je donne tous ces détails, probablement superflus, saugrenus même, pour bien montrer que ma mémoire est bonne, que ce n'est pas par une défaillance de mémoire que j'ai quasiment oublié les deux longues semaines d'existence d'avant mon retour à la vie, à ce qu'on appelle la vie. [J. Semprun L'écriture ou la vie : 43]

la présence d'un item réitéré n'en reste pas moins un peu « extra-ordinaire », le locuteur pouvant toujours faire sans, ou faire autrement. La répétition n'est jamais obligatoire et l'on pourrait tout autant trouver :

(1bis) Tout au long de son exposé - plutôt scabreux - M. Innocento n'eut qu'à se louer de son interlocuteur.

(2bis) J'aime, que dis-je ? j'idolâtre Junie

(3bis) Je donne tous ces détails, probablement superflus, saugrenus même, pour bien montrer que ma mémoire est bonne, que ce n'est pas par une défaillance de mémoire que j'ai quasiment oublié les deux longues semaines d'existence d'avant ce qu'on appelle le retour à la vie.

3 Dans tous les cas, le retour déclenche un segment second, qui s'impose comme un nouveau départ et rend provisoire la première occurrence. Même dans des cas où la 
répétition semble jouer un rôle intensif, comme en (4) et (5), le locuteur aurait pu se satisfaire des énoncés bis, (4bis et ter) ou (5bis) :

(4) Colette trouve là un moyen d'éviter le tête-à-tête avec la perspicace Sido qui veut absolument savoir si sa fille est heureuse, vraiment heureuse. [J. Chalon, Colette, l'éternelle apprentie : 62 區

(4bis) Colette trouve là un moyen d'éviter le tête-à-tête avec la perspicace Sido qui veut absolument savoir si sa fille est vraiment heureuse.

(4ter) Colette trouve là un moyen d'éviter le tête-à-tête avec la perspicace Sido qui veut absolument savoir si sa fille est heureuse, vraiment.

(5) En ce sens, et en ce sens seulement, l'adjectif est un déterminant et le nom un déterminé. [M. Wilmet, Grammaire critique du français : 109, § 126]

(5bis) En ce sens seulement l'adjectif est un déterminant et le nom un déterminé.

On peut dire que le retour à l'identique, en position seconde, met en scène une énonciation. Cette mise en scène impose toujours une Voix particulière, un locuteur qui prend en charge le retour, valide et confirme l'adéquation du mot et de son énonciation et impose un point de vue particulier venant circonscrire et redoubler l'énonciation première.

5 Sauf mention particulière - comme (2) qui met en doute l'adéquation «que dis-je aimer ? $»^{1}$ - le retour en position seconde vaut toujours pour confirmation et renvoie au centre de la notion, c'est ce que l'on a nommé par ailleurs l'opération de recentrage ${ }^{2}$ et c'est l'énonciation toute entière qui est réaffirmée :

(6) (a) l'histoire serait trop belle, ne pas déchoir, elle n'est pas donnée, c'est un masque à prendre, à prendre donc à voler, au premier occupant la beauté sans maître, le devoir d'un resplendir, depuis longtemps j'y pense, informulable,

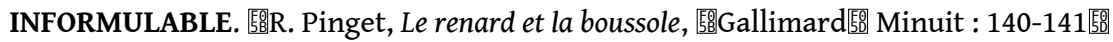

(b) (à propos d'un document papier) Il est là, LÀ ! sur la table, tu vois maintenant ?

殹Oral universitaire, 2012區

(c) Il est beau, (mais) BEAU !

\subsection{Répétitions et relances syntaxiques}

6 Le retour à l'identique permet également une meilleure appréhension de l'élaboration du discours, du discours en train de se faire. La répétition est alors un signal fort dans la progression et la hiérarchisation du discours. C'est le cas, par exemple, des relances syntaxiques, qu'on observe tout autant à l'oral qu'à l'écrit ${ }^{3}$, et pour lesquelles la répétition d'un segment, tout inutile qu'elle soit du point de vue syntaxique, sert d'autres fins $d u$ point de vue de la mise en scène énonciative et discursive. Il y a relance quand un segment d'énoncé interrompu se voit répété et complété. Richard (2002: 13) observe ce phénomène de relance: « une proposition est amorcée (E1), puis il s'y insère ce qu'on appellera un élément parenthétique (E2), enfin la répétition d'un élément du premier segment réenclenche l'enchaînement et permet l'achèvement de la proposition initiale ». Ainsi, que la relance porte sur une unité verbale (6 et 7) ou sur un segment nominal (8 et 9), dans tous les cas, le segment est répété afin d'enchaîner et d'achever une prédication jusque là restée en suspens :

(6) Une solitude profonde. Comme je me promenais sur cette croupe, j'aperçus à quelques pas d'un sentier à peine distinct, sous des buissons hérissés (à propos de buissons, le mot horridus manque dans notre langue; il dit moins qu'horrible et

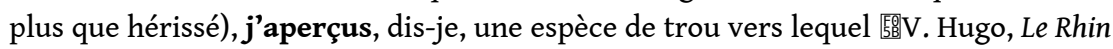
: lettres à un ami, 1842 : 316, Frantext店 
(7) INVITÉ: je pense que euh de dernière petite remarque pour euh cette cette histoire de créationnisme je pense que une des manières de répondre à euh ces inquiétudes et ces commentaires sur le créationnisme c'est de mettre l'accent dans l'enseignement scolaire sur l'histoire des sciences qui est très mal et peu enseignée.

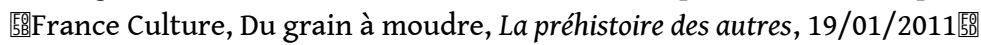

(8) Le lecteur attentif - et il doit être attentif pour tirer de sa lecture tout le bénéfice imaginable - le lecteur attentif tourne les pages du vieux livre et, parfois, revient en arrière pour s'y reconnaître dans ce désordre sanglant. [G. Duhamel, Homère au XX' siècle, Union Latine d'Éditions, Paris, 1947 : 23]

(9) ANIMATEUR: selon moi le temps et la politique on peut dire oui euh monsieur Finchelstein en parle notamment le temps et la politique sont intimement liés la chimie de la politique c'est l'urgence ceci est urgent donc prioritaire en revanche

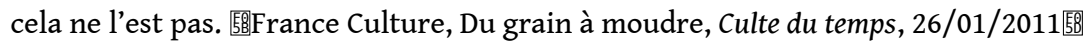

Du strict point de vue syntaxique, la seconde occurrence s'avère inutile. En (8) et (9), la référence est déjà fixée et n'a absolument pas changé ou évolué. En ce sens, le second GN n'est donc qu'un simple doublage du premier. De même en (6) et (7), on aurait pu se passer de la reprise de l'unité verbale. L'effet est ailleurs : et de nouveau dans la mise en scène d'une énonciation dédoublée. La relance syntaxique a un double effet: d'une part, elle exhibe un locuteur maitre de sa syntaxe, et d'autre part, elle est un guide pour l'interlocuteur, lui permettant d'appréhender les frontières syntaxiques d'une linéarité interrompue. La relance est en somme un signal, un signal fort, de rattrapage syntaxique (Richard \& Pennec 2009), mais conjointement, elle opère une hiérarchisation des éléments sur le plan interprétatif, elle délimite un plan principal d'un plan secondaire. En effet, tout ce qui se trouve entre le segment ainsi rappelé/réitéré et sa première occurrence se voit à présent comme mis entre parenthèses. Le raccrochage ainsi effectué réancre énonciativement et syntaxiquement l'énonciation dans l'instant présent. Tout est comme « recommencé », comme un « replay ».

8 Tout se passe comme si le retour à l'identique imposait de rejeter complètement la première énonciation et forçait à concevoir un nouveau départ, non pas seulement syntaxique mais aussi énonciatif. La répétition permet donc à la fois d'assurer/de garantir la continuité, et dans le même temps elle oblige à considérer une rupture énonciative, en tout cas un décrochement.

\section{La répétition dans l'interaction}

Ce jeu de la continuité et de la rupture se retrouve également dans les répétitions que l'on peut observer au cœur des interactions. Nous avons ainsi cherché à observer les cas de retour à l'identique dans les interactions, en prenant tout particulièrement appui sur les interactions des débats radiophoniques ${ }^{4}$. Il ne s'agit donc plus de retours chez un même locuteur, mais de reprise à l'identique entre deux locuteurs distincts : le décrochement énonciatif décrit précédemment est donc d'ores et déjà consommé ici puisqu'il s'agit bien d'observer les répétitions entre deux - ou plusieurs - énonciateurs différents.

\subsection{Une syntaxe collaborative}

10 Pourtant, si décrochement il y a, la répétition permet de gommer la rupture imposée par les différentes interactions et de poursuivre une syntaxe initiale, l'air de rien... une syntaxe que l'on peut alors dire interactive, en continu, en somme une syntaxe collaborative. 
11 L'exemple (10) viendra corroborer cette hypothèse. C'est le début de l'émission, On va déguster, sur la cuisine du Nord, l'animateur 1 présente ses invités, il introduit ici son invité 3, Alex Croquet, un boulanger :

(10) ANIMATEUR1: on va croquer de la baguette avec vous mais aussi des spécialités un peu moins nationales comme euh INVITÉ 3 : ah des spécialités un peu moins nationales comme la baguette de meule la: si j'ai ramené le pain Zébulon ANIMATEUR1 : et la faluche quand même INVITÉ 3 : et puis et puis la faluche ANIMATEUR1 : et vous avez même oublié la tarte au sucre INVITÉ 3 : et la tarte au sucre ANIMATEUR1 : en parlant de sucre on a aussi notre bec sucré sur le plateau d'On va déguster je vous demande d'accueillir Elvira Masson bonjour

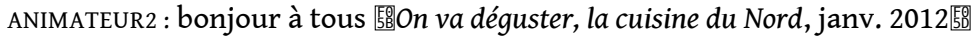

La syntaxe de l'invité 3 se construit, au fur et à mesure des interventions de l'animateur, elle s'amplifie. Ce phénomène d'amplification a déjà été observé par Claire Blanche Benveniste (鍳1997嚚 2010) dans la construction syntaxique de la langue orale; l'originalité ici réside dans le fait que l'invité reprend les éléments référentiels apportés par l'animateur et les intègre à sa syntaxe initiale par coordination successive, sans plus de commentaire.

Cette forme assez neutre de collaboration s'exerce le plus souvent de l'animateur vers l'invité ; (11) en est un autre exemple :

(11) INVITÉ : oui euh il me semble alors il faut d'abord euh préciser un petit peu la périodisation de l'élevage industriel euh il émerge en en France euh après la seconde guerre mondiale essentiellement hein euh il existe cependant à Paris au dix-neuvième siècle ce qu'on appelle la discipline de la zootechnie qui vise essentiellement à améliorer la productivité des animaux d'élevage

*ANIMATEUR1 : par la sélection par exemple

* INVITÉ: par la sélection par l'amélioration euh des races notamment euh et cette zootechnie ne s'accompagne pas euh d'un développement de l'élevage industriel

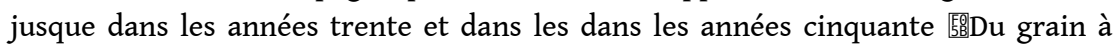
moudre, L'animal, 18/01/2011媦

ou entre deux animateurs (ici des animatrices), (12), ou entre les invités comme en (13) :

(12) ANIMATEUR1 : Jacqueline et Pablo Picasso. Il fallait sans doute un livre au moins pour parler de cette affaire. Ce livre existe il est signé Pepita Dupont, c'est publié au Cherche Midi, elle a été l'amie de Jacqueline Picasso.

ANIMATEUR2: Oui c'est une sorte de réhabilitation de cette amie. Moi j'ai lu surtout une véritable histoire d'amitié entre Pépita et Jacqueline Jacqueline qui est décédée ANIMATEUR 1 : qui s'est suicidée

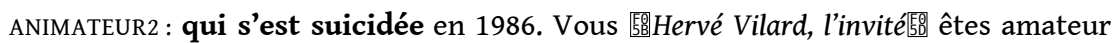
d'art on le sait. Amateur de Picasso? [5:France Bleu, Une heure et demi service compris, 2007医

La reprise-correction de l'animatrice 1 "qui s'est suicidée » est réinsérée, sans heurt dans la syntaxe de départ de l'animatrice 2 qui lui ajoute même à son tour une précision «en $1986 »$.

De même dans l'exemple (13)

(13) INVITÉ 3 : (RIRES) mais faut $d$ dans l'ensemble finalement il y a une certaine efficacité à être dans une phase euh de disons un peu un peu INVITÉ 5 : de mûrissement 
INVITÉ 3: de mûrissement ouais c'est ça de mûrissement une phase d'accumulation et qui permet ensuite de de de produire et de faire un acte qui est très créatif et qui est parfois euh un peu fulgurant si je puis dire 監France Inter, Le débat de midi, Les ados sont-ils des glandeurs ?, 22/08/2013 臨

l'acceptation pleine des mots de l'Autre (répétés une seconde fois après commentaire positif «de mûrissement oui c'est ça de mûrissement») n'en demeure pas moins immédiatement reformulée "une phase d'accumulation», et c'est sur cette ultime reformulation que la syntaxe initiale se poursuit « et qui permet ensuite..." permettant ainsi au locuteur 3 de reprendre la main.

Le ressort linguistique peut aussi être utilisé dans l'autre sens, de l'invité vers l'animateur, mais c'est plus rare ; ainsi l'exemple (14): un auditeur s'inquiète de l'impact des nanotechnologies sur les libertés individuelles:

(14) AUdITEUR: voilà / heu: j'entends parler: tous vos intervenants / mais / j'entends parler de cette technologie depuis longtemps / et je me demande si heu mal employée / elle n'est pas heu / comment vous dire // elle n'est elle n'enfreint pas la liberté de l'individu / c'est-à-dire que on peut être heu : tous sous une puce sous un un truc et devenir un numéro / vous comprenez ce que je veux dire ANIMATEUR1 : on comprend on va essayer de vous répondre si j'ai bien compris les les puces implantées

INVITÉ 1 : il s'agit de la miniaturisation voilà

ANIMATEUR1 : voilà / miniaturisation(s) implantée(s) sous la peau et cetera / et donc évidemment à terme attentatoire aux libertés dès lors que avec heu ces implantations si petites soient-ils heu soient-elles pardon permettent de / de vous situer hein 鹠Le téléphone sonne, Les nanotechnologies, 15/10/2009區

Dans sa dernière intervention, l'animateur, non seulement dit qu'il concède intérêt au mot proposé par l'invité, - cela nous est signalé par le marqueur "voilà $»^{5}$ - mais il l'intègre à sa propre structure initiale "miniaturisations implantées ", faisant d'autant plus sienne l'énonciation de l'invité qui est alors véritablement renvoyée à un « entre deux ».

Ce jeu collaboratif, acceptation pleine ou partielle des mots de l'Autre, est constant dans ces débats radiophoniques, où l'on passe une grande partie de son temps de parole à dire qu'on s'inscrit dans du déjà-dit: la répétition est à ce titre un signal majeur de construction de la co-énonciation.

\subsection{Répétition-rebond}

21 Mais prendre la parole dans ce type de débat radiophonique (comme certainement dans toute situation de communication) n'est pas anodin, ni chose facile.

De façon très ordinaire, la reprise à l'identique d'un segment, le plus souvent final, énoncé par un autre locuteur permet l'enchaînement discursif des répliques qui se répondent :

(15) ANIMATEUR1 : Et vous les 熙les passeports區 avez retrouvés depuis?

AUDITEUR: On les a pas retrouvés malheureusement on les a pas retrouvés donc on

s'est un petit peu on s'est vraiment engueulés

ANIMATEUR1 : Oui ça peut arriver ça

AUDITEUR: Oui ça peut arriver oui on s'est engueulés parce que j'étais j'ai mis tellement de cœur c'est vrai que je l'aimais tellement que / j'attendais quand même ce mariage c'était vraiment merveilleux enfin et tout s'effondrait d'un coup

(...) 
ANIMATEUR1 : Dites-moi qu'est-ce qui s'est passé depuis

AUDITEUR : Alors ce qui s'est passé depuis on a fait nos passeports j'ai refait mon

passeport alors il (...) [5:Europe 1, Ligne ouverte, Caroline Weill, 2007塸 seconde de réflexion. Il dit aussi qu'on s'inscrit dans une chronologie du discours, qu'on a bien pris acte de l'énonciation précédente, qu'on la fait sienne et qu'on la poursuit. On pourrait parler de co-énonciation, dans le sens où l'on construit ensemble un même discours. Nonobstant, ce type de reprise en écho, sans reformulation ni modalisation quelconque, ordonne une posture énonciative particulière ${ }^{6}$, que l'on pourrait dire de sous-énonciation, dans le sens de soumission à la parole de l'autre: l'interlocuteur se place dans les traces du locuteur, il admet que c'est celui-ci qui impose les règles du jeu. Dans les exemples de notre corpus, cette posture énonciative se voit confirmée par le fait que ce type de reprise est adopté le plus souvent par le locuteur-auditeur, qui se conforme ainsi à la parole de l'animateur? .

Dans ce jeu subtil de construction du débat radiophonique, les modèles de répétition utilisés par le locuteur-animateur sont très différents, plus variés également. Une des caractéristiques du discours de l'animateur, sa force énonciative aussi, c'est de savoir - et de pouvoir - rebondir sur les mots des autres locuteurs. La répétition permet alors d'extraire un élément du discours, de l'isoler comme cible, et ainsi de cadrer le champ dans lequel doit se situer le discours :

(16) INVITÉ 2 : j'ai pas assez de d'expérience dans le monde du travail mais euh par rapport à d'autres boulots que j'ai pu faire on était vraiment pressés-pressés en mode on y est tout le temps

ANIMATEUR1 : d'autres boulots vous en avez fait vous avez dix-neuf ans hein vous en avez fait beaucoup $\mathbf{d}$ 'autres boulots comme vous dites

INVITÉ 2: on j'ai fait caissier j'ai fait recruteur de donateur pour une ONG 鴡France Inter, Le débat de midi, Les ados sont-ils des glandeurs ?, 22/08/201350

Cet effet-rebond de la répétition permet à l'animateur de cibler un élément du discours pour le mettre sur le devant de la scène énonciative: soit qu'il n'était pas en position saillante jusqu'alors comme dans l'exemple précédent, soit qu'il est trop éloigné sur la chaîne énonciative comme dans l'exemple (17) ci-après où le retour s'effectue après quatre tours de paroles différents :

(17) INVITÉ 4 : donc je baigne je baigne dans ce milieu formidable et c'est vrai qu'ils ne sont pas tous comme ça mais j'aimerais revenir sur le terme petits branleurs et petits glandeurs je trouve je trouve la terminologie très intéressante à un âge où effectivement euh les les glandes sont quand même en ébullition et et et quoi qu'il en soit c'est vrai qu'ils ont besoin d'un temps euh horizontal je dirais où ils sortent régulièrement la phrase mais c'est bon je vais le faire effectivement ils vont le faire quoi qu'on leur demande par exemple range tes chaussures enlève les de la table de la cuisine par exemple euh je vais le faire mais il leur faut effectivement un temps qui n'est pas le nôtre hein il y a une temporalité qui est vraiment très spécifique aije observé

ANIMATEUR1 : à l'adolescent.

INVITÉ 4 : à l'adolescent.

INVITÉ 5 : ou alors peut-être les garçons aussi parce que vous avez quatre garçons ils sont différents des filles

INVITÉ 4 : ouais mais j'ai un panel d'observation féminin aussi

ANIMATEUR1 : en en même temps puisque vous parlez du temps je crois que c'est une phrase qui est dite par Vincent Cespedes dans votre documentaire ils ont la patience d'un temps de téléchargement d'un mp3 
INVITÉ 4 : voilà

ANIMATEUR1 : il y a à peu près deux secondes et demi

INVITÉ 4 : voilà ils font les choses très très vite aussi 鷗France Inter, Le débat de midi,

Les ados sont-ils des glandeurs?, 22/08/2013塸

26 jusqu'alors, la répétition est indispensable : sans le retour à l'identique, la cohérence du discours serait mise à mal. Difficile en effet d'imaginer l'enchainement des répliques sans le segment répété :

? (16bis) InVITÉ 2 : j'ai pas assez de d'expérience dans le monde du travail mais euh par rapport à d'autres boulots que j'ai pu faire on était vraiment pressés-pressés en mode on y est tout le temps

ANIMATEUR1 : d'autres boulots vous en avez fait vous avez dix-neuf ans hein vous en avez fait beaucoup d'autres boulots comme vous dites

? (17bis) InVITÉ 5 : ou alors peut-être les garçons aussi parce que vous avez quatre garçons ils sont différents des filles

INVITÉ 4 : ouais mais j'ai un panel d'observation féminin aussi

ANIMATEUR1 : en en même temps puisque vous parlez du temps je crois que c'est une phrase qui est dite par Vincent Cespedes dans votre documentaire ils ont la patience d'un temps de téléchargement d'un mp3 c'est elle encore qui inscrit le discours dans une continuité. En somme, c'est parce qu'elle est rappel d'un déjà-dit qu'elle assure la cohérence du discours. Double effet de cette répétition qui permet à la fois de maintenir le débat dans une continuité, d'en assurer à ce titre la cohérence, et, dans le même temps, de le recadrer, de le resserrer et ainsi de le réorienter ${ }^{8}$ - c'est ce que Rabatel nomme la sur-énonciation. N'importe quel segment du discours peut ainsi faire l'objet de ce type de d'extraction, mais pas par n'importe quel locuteur. En effet, dans le cadre des débats radiophoniques ce type de reprise a pour effet de signaler le travail d'expertise de l'animateur. C'est sur ce modèle que l'animateur construit sa posture énonciative structurante: c'est lui qui assure la cohérence du discours, c'est lui qui choisit, élit un objet de discours, c'est lui qui propulse un segment quelconque en objet du débat.

3. Répétition et transition

Le segment ainsi répété sert alors de relais, entre un avant et un après. La répétition rappelle qu'on s'inscrit dans du déjà-dit et engage un «à-dire ». Pour le locuteur elle est un moyen "économique» de rebondir et surtout de "prendre la main»; pour l'interlocuteur (ou tout autre récepteur), elle est indice de lisibilité, elle établit un lien avec un amont et oblige donc l'interprétation de continuité.

Pour autant, si le lien avec l'amont est explicite grâce au segment réitéré, c'est dans/avec l'aval qu'il faut aller chercher une interprétation différenciée de la répétition. En effet, le lien est parfois ténu et ne se maintient pas toujours. Assez étrangement, ces répétitions qui imposent un lien avec l'amont, n'engagent pas obligatoirement le locuteur dans une poursuite/continuité argumentative, mais sont au contraire parfois marques de rupture. Dans les débats radiophoniques, ces répétitions sont, à ce titre, isolables comme des lieux de transition. En nous focalisant sur les structures du type «à propos de $\mathrm{X}_{\text {répété ", nous }}$ avons ainsi observé trois modèles de transition. 


\subsection{Continuité discursive et construction de la mémoire collective} déjà-dit commencé, dans un contenu commun, et qu'il le poursuit/continue, qu'il l'abonde. En somme, la répétition maintient le lien entre ce qui précède et ce qui suit, elle est la marque d'un véritable relais thématico-référentiel. On rencontre ce mode de répétition soit dans le cadre de la distribution de la parole chez l'animateur (18a), soit dans le cadre de la prise de parole des invités (18b), on n'en a pas rencontré d'occurrences dans le discours des auditeurs :

(18) (a) ANIMATEUR1: Monsieur Bergougnoux à propos de la pénurie toujours un commentaire?

(b) INVITÉ 1 : encore un mot à propos de l'effet de serre

年 deux cas, le segment répété, "la pénurie ", "l'effet de serre », est l'objet des débats en cours. La répétition fait ici le lien entre l'amont et l'aval, elle rappelle qu'on construit, ensemble, une mémoire commune, dans une sorte de co-argumentation. Un $\mathrm{V}$ ou $\mathrm{N}$ de discours (commentaire/ encore un mot) est presque toujours présent et le segment est déplaçable :

À propos de l'effet de serre, un commentaire?

Un commentaire, à propos de l'effet de serre?

On notera cependant que la formule contenant l'item répété n'est ici nullement nécessaire, on pourrait tout autant avoir :

Mr Bergougnoux, un commentaire?

La répétition vaut donc pour rappel de l'objet commun de discours et le segment transitif impose à l'interlocuteur de s'inscrire également dans cet univers commun : on peut dire qu'il marque et qu'il impose (de) la co-énonciation. C'est toujours l'équivalent de «à propos de ce qu'on disait sur 颐LE X臨 (je vais donner mon/un Point de vue) ». La formule met ainsi en attente un nouveau point de vue à venir.

Si le segment est utilisé par un invité, c'est toujours son propre point de vue qui est ainsi mis en suspens (comme dans l'exemple 18b). Mais dans le discours de l'animateur, le segment met en attente le point de vue d'un Autre, interlocuteur direct lorsqu'il s'agit de solliciter le point de vue d'un invité (exemple 18a avec Mr Bergougnoux), ou locuteur indirect quand il s'agit de rapporter un point de vue émis par un auditeur via les réseaux sociaux, comme dans l'exemple (19) ci-après :

(19) INVITÉ 2 : je pense que les les critères d'individu xx qui sont aujourd'hui dans l'entreprise à l'œuvre qui individualisent les promotions et les salaires sont en ce sens très mauvais et on devrait revoir tous ces critères d'évaluation afin de afin de donner toute leur place aux femmes.

ANIMATEUR1: au passage à propos de ce qu'on disait sur les salaires femmes hommes l'égalité c'est pas une colle mais j'ai ce courriel de Lisa à Paris elle ne donne pas de téléphone j'ai une forte elle appelle ça comme ça une forte présomption que mon collègue masculin de même formation même âge et quelques diplômes en moins est nettement mieux payé que moi j'aimerais faire jouer l'égalité hommes femmes ou femmes hommes mais ni mon collègue ni la DRH ne veulent divulguer les salaires comment faire il y a des moyens auprès des $d u d u$

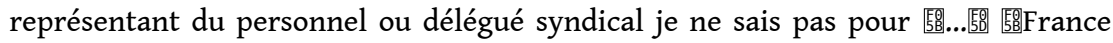
Inter, Le téléphone sonne, Égalité homme-femme, 8/03/2011臨 


\subsection{Continuité référentielle mais nouvel angle d'attaque}

Dans le second modèle, la reprise ne porte que sur le référent de $\mathrm{X}$ : la répétition de l'unité référentielle sert alors d'appui pour ouvrir un nouvel univers de discours, pas seulement pour dire autre chose de ce référent (comme ce serait le cas dans les exemples précédents) mais pour en faire autre chose. La répétition dans les exemples (16) et (17) joue bien ce rôle :

(16) INVITÉ 2 : j'ai pas assez de d'expérience dans le monde du travail mais euh par rapport à d'autres boulots que j'ai pu faire on était vraiment pressés-pressés en mode on y est tout le temps

ANIMATEUR1: d'autres boulots vous en avez fait vous avez dix-neuf ans hein vous en avez fait beaucoup d'autres boulots comme vous dites

INVITÉ 2: on j'ai fait caissier j'ai fait recruteur de donateur pour une ONG 喕France Inter, Le débat de midi, Les ados sont-ils des glandeurs?, 22/08/2013 區

(17) ANimATEUR1: en en même temps puisque vous parlez du temps je crois que c'est une phrase qui est dite par Vincent Cespedes dans votre documentaire ils ont la patience d'un temps de téléchargement d'un mp3

Le locuteur-animateur met à profit (profite de) l'énonciation d'un objet de discours pour ouvrir un nouveau débat, disons pour en faire un nouvel objet de débat. La répétition n'est pas sans rappeler les structures cadratives (au sens de Charolles) « à propos de X, Quant à X, au sujet de X» étudiées notamment par Porhiel (2001). Pourtant, ce type d'introducteur serait impossible en (16) * «à propos d'autres boulots», le cadratif n'acceptant que les référents définis, «à propos des boulots, de ces boulots, de vos boulots ». L'intervention (17) pourrait, elle, intégrer la séquence "à propos du temps ». Mais on n'en a pas rencontré dans nos corpus radiophoniques'. En revanche, une recherche sur Frantext en fait apparaitre dans les journaux ou la correspondance, c'est-àdire dans des situations d'interaction feinte :

(20) Parmi ceux que j'ai vus ces jours-ci il y a François Périer, Yves Robert. Mais Maria Schell (ou Shell) emmerde Clément à longueur de journée. Tiens, à propos de Clément, c'est un drôle d'intuitif. Parce qu'il est revenu sur ce qu'il m'a dit il y a

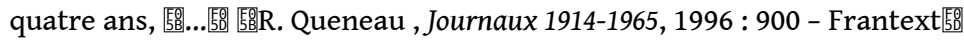

(21) Votre ami Larbaud (qui publie dans la N. R. F. un roman bien intéressant) m'a évité deux gros contresens. À propos de la N. R. F. vous devriez faire des observations au service d'expédition. Les trois abonnés que je vous ai procurés à

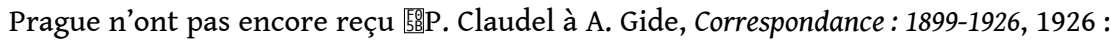
134 - Frantext医

Cette fois la présence du cadratif est obligatoire, la répétition seule n'est pas envisageable, elle n'est pas suffisante pour marquer le jeu du dédoublement énonciatif. Le décrochement est ici signalé par le cadratif (à propos de), et c'est un nouvel énonciateur qui prend la parole ${ }^{10}$. Le mobile de sa prise de parole, c'est le référent $\mathrm{X}$ énoncé précédemment. L'extraction ainsi effectuée proclame l'à-propos de l'énonciation du référent, lui concède intérêt, et permet au locuteur de re-cadrer le discours sur ce référent précisément.

C'est toujours l'équivalent de " puisque vous parlez de 嚂leX監 (j'ai quelque chose d'autre à dire à propos de ce référent) » mais c'est bien toujours de ce référent qu'on traite dans le contexte ultérieur, sous un autre angle (plus qu'un nouveau point de vue, il s'agit véritablement d'un nouvel angle d'attaque). Les exemples (16) et (17), sans présenter le 
segment «à propos de $\mathrm{X}$ », imposent néanmoins cette interprétation de recadrage par décrochement.

\subsection{Continuité notionnelle et détournement argumentatif}

Dans le dernier modèle, la reprise ne porte ni sur le contenu ni sur le référent :

(10) ANIMATEUR1 : et vous avez même oublié la tarte au sucre INVITÉ 3 : et la tarte au sucre.

ANIMATEUR1 : en parlant de sucre on a aussi notre bec sucré sur le plateau d'On va

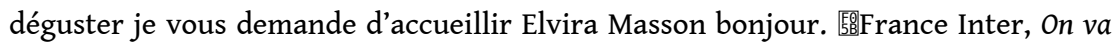
déguster, 15/10/2012菴

(22) INVITÉ 1 : oui en effet l'objectif est non seulement d'informer mais d'écouter tout le monde / alors bien sûr heu des gens qui sont des spécialistes de la question des chercheurs des industriels qui produisent des nanoparticules des industriels qui les utilisent / mais aussi heu : ceux qui ont des appréhensions / concernant la santé l'environnement / concernant aussi heu : les frontières

ANIMATEUR1: oui

INVITÉ 1 : entre la nanotechnologie et la biotechnologie et finalement les questions de la condition humaine

ANIMATEUR1 : à propos de heu à propos d'appréhension monsieur Bergougnoux et on en parlera avec les autres invités que j'ai : présentés j'ai déjà / heu des questions d'auditeurs et des courriels heu d'internautes qui trouvent que / comment dire ce débat arrive un peu tard dès lors que la science que l'industrie heu : utilisent déjà au : en tout ou partie les les nanotechnologies qui sont expérimentées voir heu / utilisées à plus large échelle / que que répondre à ces internautes et ces auditeurs

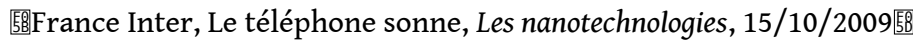

Ces occurrences de répétition se distinguent des précédentes sur deux points au moins. Du point de vue de la forme, l'élément repris ici est, au sens propre, un «matériel lexical formel » il ne s'agit pas ici d'un élément référentiel, ni d'ailleurs de l'autonyme. Pour Péroz (2003 : 416) « la perte des déterminations marque bien que ce n'est pas la reprise du contenu de Yo qui importe mais le fait qu'il s'agit d'une mention de la notion de $Y$ », cette non détermination donne directement accès à la notion $\mathrm{X}(\mathrm{N}$ sans déterminant, $\mathrm{V}$ à l'infinitif pour l'essentiel). Par ailleurs, le retour ne peut se faire sans l'insertion dans une structure cadrative " en parlant de X / à propos de X ", la répétition, seule, n'est pas suffisante $^{11}$.

On peut parler, à la suite de Porhiel (2001) et Péroz (2003), de coup de force énonciatif: en effet «à propos de X» impose un nouveau programme discursif - c'est la valeur du cadratif - mais on peut aussi parler de coup de force argumentatif : le retour en position non déterminé n'est plus qu'un alibi - Péroz (2003) parle de prétexte - pour pouvoir « reprendre la main » et n'oblige aucun lien entre l'amont et l'aval. Seule la forme répétée fait lien, dit un lien, c'est une sorte de marche-pied, qui permet au locuteur de détourner le débat sur un autre/nouvel objet du discours.

En somme, avec déterminant, l'unité référentielle répétée sera l'objet de la prédication ultérieure, on continue de parler d'un élément introduit précédemment par un autre énonciateur. Sans déterminant, il n'y a plus que la forme, qu'une forme, qui retienne l'attention du locuteur, qui se désolidarise un peu plus encore du discours antérieur.

Combettes (2005) rappelle que :

Quel que soit le type de discours, les expressions cadratives ont pour fonction essentielle de marquer les articulations du texte. Jouant en ce sens un rôle 
identique à celui des syntagmes topicalisés, qui signalent la réactivation d'un référent ou l'introduction d'un nouveau référent dans le flux discursif, elles délimitent des portions de texte qui se définissent par opposition à leur entourage contextuel. Si elles signalent l'ouverture d'un nouvel espace, elles sont également, dans le fil du texte, des signaux de fermeture. (Combettes 2005 : 41-42) pertinentes. En effet, utiliser la structure « à propos de X » n'a pas seulement pour effet de "gommer l'effet coq-à-l'âne» comme le propose Péroz (2003: 417) entre les deux prédications ainsi reliées, elle permet surtout au locuteur, comme on l'a dit, de reprendre la main, de se ré-approprier la parole, et de ré-orienter le discours comme bon lui semble, le principe de coopération étant respecté a minima par le retour de X. Dans le même temps, elle interdit à l'interlocuteur de poursuivre l'argumentation première. Ce type de transition impose qu'on change de cap, elle impose aussi la fermeture de l'argumentation antérieure: impossible pour l'interlocuteur de reprendre, d'enchaîner sur l'argumentation antérieure sans y être (d'une façon ou d'une autre) explicitement autorisé :

(24) Président - Je suis maintenant à votre écoute, ces réunions doivent servir le débat

(Silence dans la salle)

Vice-Présidente 1 - À propos de réunion, ça vous laissera le temps de réfléchir à vos questions, je profite pour vous rappeler que je viendrai visiter les personnels

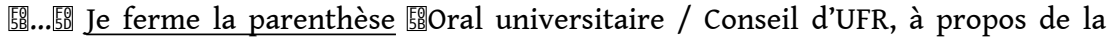
fusion Rennes1/Rennes2, nov. 2013医

En (24), le décrochage n'est plus seulement énonciatif mais aussi discursif et argumentatif. Sans la séquence "je ferme la parenthèse", il serait difficile pour les interlocuteurs de ne pas enchaîner sur le discours de la Vice-Présidente. C'est donc cette

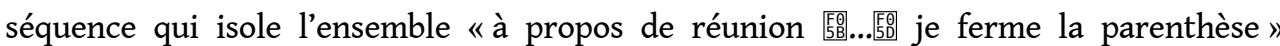
comme extérieure au débat. Elle a pour effet de clore la séquence énonciative de la VicePrésidente et de rétablir le cours initial du débat. Les interlocuteurs sont alors autorisés à répondre au Président.

\section{Conclusion}

Toutes les répétitions s'inscrivent et signalent une forme de chronologie du discours, elles sont des repères, des indices que quelque chose avance. La répétition est toujours mise en scène d'une Voix particulière, elle oblige à interpréter de la continuité (l'item $x$ répété faisant nécessairement office de lien avec une énonciation précédente), mais oblige en même temps à interpréter un décrochement. Décrochement qui peut aller jusqu'à la rupture d'argumentation, le retour-alibi de X, débarrassé de ses déterminants, signale alors une assez mauvaise transition, abrupte, mais qui doit se dire et qui de fait, ne laisse pas l'interlocuteur sans instruction particulière. Dans les débats radiophoniques, elle met fin à une argumentation, voire elle en nie toute pertinence : on peut dire qu'elle gomme la valeur argumentative de ce qui précède. Dans tous les cas, ce type de reprise/ transition, de reprise-alibi fait apparaître un locuteur maître du jeu, et d'ailleurs dans nos corpus médiatiques, seul l'animateur s'autorise ce type de rupture d'argumentation.

Les interactions radiophoniques sont un lieu privilégié pour l'analyse conjointe des formes de répétitions et des postures énonciatives qui y sont associées. Nous avons ainsi observé que les auditeurs, les invités ou les animateurs n'utilisent pas les mêmes modèles 
de répétition, et réciproquement, c'est l'analyse de ces structures qui permet d'identifier le type de locuteur. Une étude approfondie devrait participer à affiner une typologie des postures énonciatives.

Chemin faisant, la réflexion pose la question des moyens linguistiques utilisés pour faire transition, pour dire la transition, on pense à tous les segments du type " pour faire une transition, pour changer de sujet, passons maintenant à », et l'incroyable «sans transition» - qui ne sert qu'à ça - tous des segments qui n'imposent pas, voire n'autorisent pas la répétition, contrairement aux cas envisagés ici.

\section{BIBLIOGRAPHY}

Authier-Revuz, J., (1995), Ces mots qui ne vont pas de soi. Boucles réflexives et non-coïncidence du dire. 2 tomes, Coll. «Sciences du langage », Paris : Larousse.

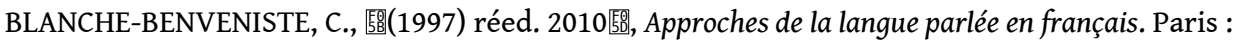
Ophrys.

CHAROLLES, M., (2003), « De la topicalité des adverbiaux détachés en tête de phrase ». Travaux de linguistique, 47, p. 11-49.

CHAROLLES, M. \& VIGIER, D., (2005), « Les adverbiaux cadratifs en position préverbale : portée cadrative et organisation du discours ». Langue française, 148, p. 9-39.

Combettes, B., (2005), « Les constructions détachées comme cadres du discours ». Langue française , 148, p. 31-44.

DELAHAIE, J. (à par.), «Voilà c'est ça, voilà c'est tout, et puis voilà. Interprétation syntaxique et sémantique des emplois en conversation de voilà ». In É. Richard \& S. Oriez (éds), (à par.) Des organisations dynamiques de l'oral. Berne : Peter Lang.

MOLINIER, C., (2003), « Connecteurs et marqueurs énonciatifs : les compléments figés formés à partir du nom propos ». Linguisticae Investigationes, 26 :1, p. 15-33.

NDIONE, A., (2013), Contribution à une étude de la différence entre la réduplication et la répétition en français et en wolof. Thèse. Université François Rabelais Tours.

NOAILLY, M. \& RICHARD, É. (sous presse), « Mécanique et dynamique de la répétition : figures de rhétorique et usages spontanés ». In J. Delaplace, L'art de répéter. Rennes : Presses Universitaires de Rennes.

PEROZ, P., (2003), « Sur à propos de et la valeur d'à propos de sur ». In B. Combettes, C. Schnedecker \& A. Theissen (éds), Ordre et distinction dans la langue et le discours. Paris : $\mathrm{H}$. Champion, p. 409-424.

Porhiel, S., (2001), «Au sujet de et à propos de - une analyse lexicographique, discursive et linguistique ». Travaux de linguistique, 42-43, p. 171-181.

PORHIEL, S., (2005), « Les séquences thématiques ». Langue française, 148, p. 111-126.

PREVOST, S., (2003), « Les compléments spatiaux : du topique au focus en passant par les cadres ». Travaux de linguistique, 47, p. 51-77. 


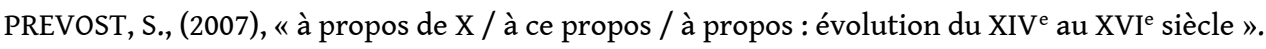
Langue française, 156, p. 108-126.

RABATEL, A., (2004), « Déséquilibres interactionnels et cognitifs, postures énonciatives et coconstruction des savoirs : co-énonciateurs, sur-énonciateurs et archi-énonciateurs ». In A. Rabatel (dir.), Interactions orales en contexte didactique. Mieux (se) comprendre pour mieux (se) parler et pour mieux (s') apprendre. Lyon : P.U. de Lyon, p. 29-66.

RABATEL, A., (2007), « Les enjeux des postures énonciatives et leur utilisation en didactique », Éducation et didactique, vol. 1, n², p. 89-116.

RABATEL, A., (2008) Homo narrans. Pour une analyse énonciative et interactionnelle du récit. Tome 1\&2. Limoges : Éditions Lambert-Lucas.

RICHARD, É., (1999), « Félix est beau, mais beau !: du dit au dire ». Revue de Sémantique et Pragmatique, 5, p. 111-118.

RICHARD, É., (2000), La répétition : syntaxe et interprétation. Thèse de doctorat, Université de Bretagne Occidentale, Brest.

RICHARD, É., (2002), « La répétition comme relance syntaxique ». l'Information Grammaticale, 92, p. 13-18.

RICHARD, É., (2008), « Mais que corrige la reformulation ? Le cas de structures avec la réitération d'un même lexème ». In M.-C. Le Bot, M. Schuwer \& E. Richard (éds) La reformulation. Marqueurs linguistiques - Stratégies énonciatives. Rennes : PUR, coll. Rivages linguistiques, p. 147-154.

RICHARD, é., (2014), Parcours de la répétition : un cercle dynamique. Synthèse HDR, Strasbourg.

RICHARD, É. \& PENNEC, B., (2009), « La relance syntaxique : le rattrapage d'une rupture ?». In I. Evrard \& al. (éds), Représentation du sens linguistique 3. Bruxelles, Belgique : De Boeck-Duculot, p. 241-251.

RICHARD, É. \& DOQUET, C., (dir.), (2012), Les représentations de l'oral chez J.-L. Lagarce - Continuité, discontinuité, reprise. Louvain-la-Neuve, Belgique : Academia Bruylant.

RICHARD, É., Wojciechowska, B., Le Bot, M.-C., Wilczyńska, W., Gorecka, J., Bourvon, M.-F., (sous presse), « La langue du débat radiophonique : un défi pour l'enseignement-apprentissage du FLE au niveau avancé ». In C. Martinot \& A. Pégaz Paquet (éds), Innovations didactiques en français langue étrangère. CRL éditions.

RICHARD, É. \& ORIEZ, S. (éds), (à par.) Des organisations dynamiques de l'oral. Berne : Peter Lang.

\section{NOTES}

1. On pourrait aussi avoir des occurrences du type « enfin, aimer, ce n'est pas exactement le terme » ou qui viennent clairement nier l'adéquation « non, pas aimer / aimer n'est pas le terme ».

2. Sur ce point voir par exemple, Richard É. (1999) et (2000), (2014). On peut également reporter le lecteur à la récente thèse de Ndione A. (2013).

3. On peut dire que c'est un moyen linguistique privilégié pour représenter l'oral dans les œuvres littéraires - Richard É. et Doquet C. (dir.), (2012) ; Noailly M. et Richard É. (sous presse).

4. Émissions radiophoniques de type Le téléphone sonne, qui font intervenir trois locuteurs distincts : un (ou des) animateur(s), des invités en studio et des auditeurs qui se 
manifestent en direct par téléphone, ou via les réseaux sociaux (courriels, SMS, tweet, etc.).

5. La répétition en écho du marqueur « voilà » impose également des commentaires sur la (co-) construction du déjà-connu dans le discours. En effet, sa présence à la finale de l'invité 1 impose le concept « de la miniaturisation » comme du déjà-connu, seulement rappelé par le locuteur. La force de l'animateur c'est de rebondir sur cet argument d'autorité : son « voilà » initial confirme et valide l'existence du concept mais l'animateur l'intègre dans un paradigme plus vaste qu'il initie alors (miniaturisation(s) implantée(s) sous la peau et cetera), la « fermeture » de la liste par « et cetera » imposant aussi une certaine forme de connivence entre les interlocuteurs et de façon plus large encore entre les débatteurs et les auditeurs (sur ce point, on renvoie à Richard É. et al. (sous presse). Et sur " voilà », on renvoie notamment aux travaux de Delahaie J. (à par.).

6. Nous prenons appui sur les notions de postures énonciatives telles que proposées par A. Rabatel (2004-2007-2008, par exemple). L'auteur définit la co-énonciation comme la coconstruction d'un PDV commun et partagé ; la sous-énonciation, comme la construction inégale d'un PDV dominé ; et la sur-énonciation comme la construction inégale d'un point PDV surplombant. Ces trois postures permettant de « rendre compte plus finement des continuums entre consensus et dissensus, tout en articulant la construction du dire, au fil $\mathrm{du}$ discours, avec les dimensions cognitives et interactionnelles dont il est possible de retrouver les traces par l'analyse de la l'énonciation et de la référenciation » (2007: 89).

7. À plusieurs reprise, A. Rabatel met en garde contre un usage abusif et déterministe des postures en co-, sur- et sous-énonciation : « Sur le plan des places - pas plus que sur celui des activités -, il n'y a pas de corrélation entre coénonciation et égalité, surénonciation et

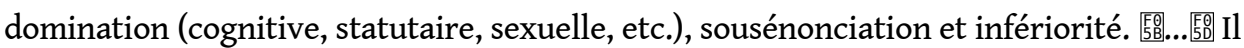
serait donc réducteur de réifier les postures en fonction des places ou des stratégies voire des situations ou des intentions. En revanche, la contribution des postures à la dynamique des places est précieuse » $(2007: 78)$. C'est dans cette dynamique des places que l'étude des formes de répétitions nous paraît pertinente : traces linguistiques d'une posture énonciative qui se construit dans et par le discours, les schémas de répétition ici étudiés mettent corrélativement au jour des instances énonciatrices (des locuteurs) qui se soumettent bon gré mal gré à la scénographie imposée par les interactions radiophoniques.

8. C'est l'effet topique-focalisation de Sylvie Porhiel (2001, 2006, par exemple) ou de Sophie Prévost (2003) à la différence qu'ici les éléments extraits sont des segments répétés qui à ce titre re-viennent sur le devant de la scène.

9. Ce qu'on n'explique pas encore et qui méritera d'être étudié plus avant. 10. En (20), le décrochement se voit initié par le marqueur «Tiens », indice supplémentaire à la fois d'une interaction feinte dans ce genre littéraire et du décrochement énonciatif.

11. L'inverse n'étant pas vrai à propos se suffit à lui-même, mais pas en parlant :

(10') INVITÉ 3 : et la tarte au sucre.

ANIMATEUR1 : à propos on a aussi notre bec sucré sur le plateau

?? (10") INVITÉ 3 : et la tarte au sucre.

ANIMATEUR1 : en parlant on a aussi notre bec sucré sur le plateau 


\section{ABSTRACTS}

Lexical repetition is defined as the occurrence of a given lexical item a second time. This study deals with the notions of continuity and rupture that are inherent to repetition. Thus, after observing certain patterns of repetition by the same speaker, we analyze here the repetitions that occur in the interactions characteristic of radio call-in discussions. The study reveals repetition patterns which recall something that has already been said (" déjà-dit ») and pave the

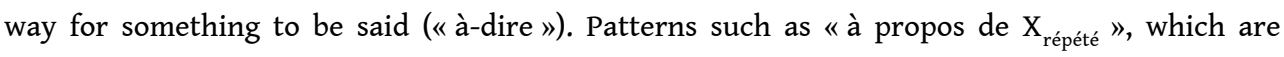
prototypical of transition, appear to also be valuable objects to study utterers' postures in radio debates.

Considérant la répétition lexicale comme le retour à l'identique, en position seconde, d'un même item lexical, l'article interroge les notions de continuité et de rupture qui lui sont inhérentes. Ainsi, après avoir observé certaines formes de répétition chez un même locuteur, l'article propose d'analyser plus précisément les répétitions qui interviennent dans les interactions des débats radiophoniques. Il met en outre au jour des schémas de répétitions qui rappellent du "déjà-dit » et engagent un à-dire. Lieux de transition, les schémas du type "à propos de $\mathrm{X}_{\text {répété " }}$ sont également des lieux privilégiés pour étudier les postures énonciatives différenciées qui se dégagent dans les échanges radiophoniques.

INDEX

Mots-clés: Répétition, Syntaxe, Débat, Transition, Argumentation

Keywords: Syntax, Argumentative dimension

\section{AUTHOR}

\section{ÉLISABETH RICHARD}

Université Rennes 2LIDILE EA 3874 